\section{Experimental Evidence regarding the Field of the Deuteron}

A METHOD for determining the field surrounding nuclei is to scatter charged particles by the nuclei in question. If the field were of the Coulomb type, the yield of nuclei projected in a given direction under the bombardment of $\alpha$-particles would be proportional to $1 / V^{4}$, where $V$ is the velocity of the incident $\alpha$-particles. Any deviation from the Coulomb field will manifest itself in a deviation from this relation. The experiments of Chadwick and Bieler ${ }^{1}$ have shown that such anomalous scattering is very clearly evident in collisions between $\alpha$-particles and protons for $\alpha$-particle velocities corresponding to ranges greater than about $2 \mathrm{~cm}$.

We have made similar experiments to determine the range at which anomalous scattering begins for $\alpha$-particle impacts (1) with protons, (2) with deuterons. Our results for protons confirm the work of Chadwick and Bieler and show detectable anomalous scattering at $1.7 \mathrm{~cm}$. $\alpha$-particle range for head-on collisions; experiments at a greater angle showed that the anomaly occurs at a larger range but for the same distance of closest approach. The yield curves for deuterons are of the same form as for protons, as suggested by Rutherford and Kempton ${ }^{2}$, but the anomaly begins at a lower $\alpha$-particle range, namely, $1.45 \mathrm{~cm}$. for head-on collisions.

If one calculates the distance of closest approach for the two cases, taking account of the different masses of the projected particles, one finds that the deviation from the Coulomb field occurs at $4.6 \times 10^{-13}$ $\mathrm{cm}$. for protons and $3 \cdot 1 \times 10^{-13} \mathrm{~cm}$. for deuterons. It is of interest that the attractive nuclear field extends farther in the case of the proton than it does in the case of the deuteron. If known corresponding radii for higher elements are plotted against $Z$, then it is the proton which lies off the extrapolated curve, the deuteron being more nearly regular.

$$
\begin{aligned}
& \text { Yale University. } \\
& \text { Feb. } 1 . \\
& \text { H. Margenau. }
\end{aligned}
$$$$
\text { E. Pollard }
$$

\section{$\beta$-Spectra of Some Radioactive Elements}

WE have investigated the $\beta$-spectra of radioactive elements that are obtained by bombarding chlorine, bromine and iodine with neutrons. As E. Fermi, E. Amaldi, O. D'Agostino, E. Rasetti and E. Segré1 have shown, in all these cases radioactive isotopes of the bombarded elements are formed.

A glass tube containing beryllium and 200 millicuries of radon was used as the source of neutrons. Surrounding the source with substances rich in hydrogen ${ }^{2}$ highly increases in the case of bromine and iodine the probability of formation of the radioactive nuclei, and in the case of chlorine gives a marked effect ${ }^{3}$. Therefore we immersed the source, together with the sample to be irradiated, in a container filled with water.

Radioactive chlorine was observed by using carbon tetrachloride, and radioactive bromine and iodine were obtained from ethyl bromide and methyl iodide, the active atoms being separated from the irradiated substance, as suggested by Szilard and Chalmers ${ }^{4}$, in the form of a thin layer of the corresponding silver compound.
The energy distribution of the electrons emitted was measured by the magnetic analysis method with

\begin{tabular}{|c|c|c|c|c|}
\hline $\begin{array}{l}\text { Irradiated } \\
\text { substance }\end{array}$ & $\begin{array}{l}\text { Radioactive } \\
\text { substance }\end{array}$ & Period & $\begin{array}{l}\text { Limit of the } \\
\text { spectrum }\end{array}$ & $\begin{array}{l}\text { Maximum of } \\
\text { the spectrum }\end{array}$ \\
\hline $\begin{array}{l}\text { Chlorine } \\
\text { Bromine } \\
\text { Bromine } \\
\text { Iodine }\end{array}$ & $\begin{array}{l}\mathrm{Cl}^{36} \\
\mathrm{Br}^{80} \\
\mathrm{Br}^{82} \\
\mathrm{I}^{128}\end{array}$ & $\begin{array}{l}50 \mathrm{~min} . \\
30 \mathrm{~min} . \\
6 \mathrm{hr} . \\
30 \mathrm{~min} .\end{array}$ & $\begin{array}{l}2,050 \pm 100 \mathrm{kv} . \\
2,100 \pm 100 \mathrm{kv} . \\
2,100 \pm 100 \mathrm{kv} \text {. } \\
2,100 \pm 100 \mathrm{kv} .\end{array}$ & $\begin{aligned} & \overrightarrow{500 \mathrm{kv} .} \\
< & 300 \mathrm{kv} . \\
& 500 \mathrm{kv} .\end{aligned}$ \\
\hline
\end{tabular}
two Geiger-Müller counters already described ${ }^{5}$. The results obtained are shown in the last two columns of the following table:

So far as the accuracy of our measurements goes, all the elements investigated have the same spectral limits. Furthermore, $\mathrm{Br}^{80}$ and $\mathrm{I}^{128}$ have not only the same periods and spectral limits, but also the same shape of the spectral curve, analogous to that of radium E. By comparing the spectral limits obtained here with the masses of the nuclei involved in the nuclear reactions, emission of hard $\gamma$-rays is to be expected.
A. I. Alichanow.
A. I. Alichanian.
B. S. Dželepow.

Physical Technical Institute,

Leningrad.

Jan. 22.

${ }^{1}$ E. Fermi, E. Amaldi, O. D'Agostino, E. Rasetti and E. Segré, Proc. Roy. Soc., A, 146, $483 ; 1934$.

E. Fermi, Aegré, La Ricerca Scientifica, V, 2 ; 1934.

3 E. Amaldi, O. D'Agostino, E. Segré, La Ricerca Scientifica, V, 2 934.

Szilard and Chalmers, NATURE, 134, 462; 1934

A. J. Alichanow, A. I. Alichanian and B. S. Dželepow, NaTURE, 133,$950 ; 1934$.

\section{Ionosphere Measurements during the Partial Eclipse of the Sun of February 3, 1935}

PULSE measurements were made at Deal, N.J., during the solar eclipse of February 3, 1935. This eclipse began at 10.28 a.m. and ended at 12.32 p.m. with a maximum effect at the ground at Deal of approximately 40 per cent magnitude at 11.30 a.m. (E.S.T.).

The critical ionisation frequencies for the $E, M^{1}$ and $F_{2}$ regions were measured on the day of the eclipse from 8.30 a.m. to 2.00 p.m. as well as on the two following days.

Our results show that the eclipse was accompanied by a decrease in the maximum ionic density of $20-25$ per cent in all three regions, and that the minimum ionisation occurred at or very shortly after the eclipse maximum. The percentage decrease was progressively greater from the lowest to the highest region, being approximately 20 per cent for the $E$ region, 22 per cent for the $M$ region and 25 per cent for the $F_{2}$ region. A progressive increase of this order is to be expected from the fact that the eclipse had a magnitude of 40 per cent at the ground and approximately 43 per cent in the $F_{2}$ region $(250 \mathrm{~km}$. over Deal). These magnitudes are in terms of the sun's diameter, which for this eclipse means an eclipsed area of 29 and 31 per cent, respectively.

This decrease in ionic density may be compared to a 50-60 per cent decrease in the $E$ region ionisation during the eclipse of August 31, 1932, when the eclipse magnitude was 95-100 per cent.

A number of observers ${ }^{2}$ who made measurements during the 1932 eclipse agreed that while there may have been an eclipse effect in the $F_{2}$ region, it could not be definitely attributed to the eclipse in view of 